Case Report

\title{
Diagnosis and Treatment of Psychiatric Comorbidity in a Patient with Charles Bonnet Syndrome
}

\author{
Jasper J. Chen ${ }^{1,2}$ \\ ${ }^{1}$ Behavioral Health Services, Cheyenne Regional Medical Center, Cheyenne, WY 82001, USA \\ ${ }^{2}$ Department of Psychiatry, Geisel School of Medicine at Dartmouth College and Dartmouth-Hitchcock Epilepsy Center at \\ Dartmouth-Hitchcock Medical Center, Lebanon, NH 03756, USA
}

Correspondence should be addressed to Jasper J. Chen; jjchencheyenne@gmail.com

Received 27 June 2014; Revised 7 October 2014; Accepted 17 October 2014; Published 6 November 2014

Academic Editor: Thomas Hyphantis

Copyright (C) 2014 Jasper J. Chen. This is an open access article distributed under the Creative Commons Attribution License, which permits unrestricted use, distribution, and reproduction in any medium, provided the original work is properly cited.

Background. A significant proportion of patients with neurological disorders may have comorbid psychiatric symptomology, which may be managed by primary outpatient neurologists. Referral to their psychiatric colleagues is mediated by available consultationliaison units and according to clinical opinion. Aims of Case Report. We present the case of a patient whose initial referral to epilepsy clinic led to a workup which ultimately diagnosed her with nonepileptic seizures (NES). In the course of her follow-up, she developed intractable headaches, and worsening mood symptoms and eventually exhibited Psychotic Features for which psychiatry became coinvolved in her care. Major Depression with Psychotic Features and Charles Bonnet syndrome were considered as a likely comorbid diagnoses. Her pharmacologic management on venlafaxine and quetiapine eventually caused substantial amelioration of her psychiatric symptomology as longitudinally followed by PHQ-9 and GAD-7 scores. Conclusion. Optimal evaluation and management of mental illness in patients with complex neurologic symptomology may require independent evaluation and treatment by psychiatrists when clinically appropriate.

\section{Background}

Charles Bonnet syndrome (CBS) is principally characterized by complex visual hallucinations and ocular pathology causing vision loss [1]. Other characteristics include insight into the unreality of the perceptions, absence of mental disorders, and preserved cognitive status [2]. Cognitive impairment, stroke, or early Alzheimer's disease may be predisposing conditions. Furthermore, albeit the hallucinations being classically purely visual [3], a small minority of patients with CBS have reported concomitant auditory hallucinations. Patients with CBS, especially when having comorbid psychiatric symptomology and complex medical histories, may make diagnosis and treatment challenging. They may also often encounter significant mood symptoms more optimally addressed by psychiatrists.

\section{Case Report}

We present the case of a 66-year-old woman referred originally to Dartmouth-Hitchcock Epilepsy Center in 2010 for seizure disorder. In her 20s, she was involved in a motorcycle collision for which she was hospitalized for one week. No known traumatic brain injury (TBI) was diagnosed. Then she developed migraine headaches later, becoming more severe in her 30s. Also in her 30s and 40s, she developed fainting episodes. When she was standing for a long time, she would tumble down and get up. If she stood quickly, she would lose her vision. Those were diagnosed as syncope and were present all her life. It also seemed to run in her family.

Then, in 2007, she developed what she thought were seizures. They occurred when she was lying in bed. She would wake up and shake in the middle of the night. She would not lose consciousness. They were diagnosed as nonepileptic seizures (NES). Of note, the patient denied having any history of trauma, stressful life events, or other current stressors that might have triggered spells.

The patient initiated levetiracetam but nevertheless continued having events where she started whole-body shaking, lasting 5 to 10 minutes. She described having two recent events where she only had shaking of her right hand, arm, and face. After this event, her eyes were closed and she was 
very tired and slept for several hours. She had two of those right-sided events. All other events previously have included whole body shaking.

In the summer of 2008, she had an event where she was walking to the bathroom at night, she crashed down on the floor, had raccoon eyes and bruises, and was hospitalized elsewhere. There she had tilt table testing, which was positive and she was diagnosed with syncope; however, she was also noted to be hypertensive. She was not started on any medications during that outside hospitalization but was advised to sit at the edge of the bed and stand up slowly.

She also complained of having comprehension difficulties and poor memory, citing difficulties doing the laundry. At times, she was incapable of using a coffee maker that she has used for a long time and her husband confirmed that those events were more frequently occurring. She had not been sleeping very well and had difficulties concentrating. She denied feeling depressed. Physical exam found no cogwheeling but falling diffusely with positive Romberg's and her eyes closed. She also had difficulties performing tandem gait but was able to walk on her heels and toes. The plan was for her to be admitted for video EEG (VEEG) monitoring and to have neuropsychological testing.

VEEG monitoring six weeks later in April 2010 captured multiple NES but no episodes with ictal correlates. She was discharged with outpatient follow-up outside our institution. A year later, in March of 2011, she re-presented with the chief complaints of headaches and did not have any significant NES spells. At this time, it was noted that while she did not have psychiatric care, she was taking both citalopram and clonazepam as prescribed by her primary care physician (PCP).

Half a year later in September 2011, the patient was seen with the chief complaint of headache as well as worsening depth perception. She was prescribed topiramate for her headaches and considered usage of amitriptyline, although patient was concerned about possibility of weight gain as had happened in the past. The patient was counseled on nonpharmacologic treatments of both anxiety and sleep hygiene. Nine months later in June 2012, the patient first described having more frequent falls as well as worsened headaches. For her symptoms, she was increased on topiramate prescribed hydroxyzine for moderate headaches and zolmatriptan for more severe headaches. The patient did not exhibit significant clinical improvement.

Then, in May 2013, the patient and her family described having hallucinations nearly every night, which consisted of the patient's witnessing other people talking to each other, but never to her directly. These people never talked to her or told her to do things. She denied these as the typical auditory hallucinations worrisome of primary thought disorders consisting of running commentary about the patient's own behavior. The auditory hallucinations were also never present in the absence of visual hallucinations. The visual hallucinations were nonhostile, but the patient's emotions associated with them were of fear due to uncertainty of the intentions of the people she was experiencing. However, she "knew that these people weren't really there" and that when she "turned on the lights, they would disappear." She also described episodes where she was confused and had wandered about. This happened in late February and again early March 2013. Although she had been living in the same house for 37 years, it did not feel like the same house to her.

Visual symptoms included "seeing blue spots under my eyes on the left as well as on my hands." Fundoscopic exam revealed floaters. The patient reported seeing cracks in the wall when looking at a white wall. The patient's husband reported that "she is becoming very forgetful." These hallucinations were thought to be associated with nortriptyline, and dosage was reduced to $10 \mathrm{mg} \mathrm{PO}$ at night. It was first thought and documented at the end of May 2013 that the patient may have a neurodegenerative disease especially given significant atrophy of her frontal lobes as evidenced on magnetic resonance imaging (MRI). Four months later, her falls and headaches continued, although now she was "hearing voices that are not threatening." The goal had been to decrease her nightly clonazepam dosage to see if this would allow hallucinations to improve. Of all her concerns, her headaches were causing the most misery.

At this point, referral to embedded psychiatric clinician within the neurology outpatient clinic occurred. The patient met our newly established quality improvement referral criteria by scoring above a certain threshold on depression screening. She was seen initially at the end of November, 2013. In addition to confirming her history above, it was found that family history was significant for early-onset Alzheimer's disease in the patient's brother. Physical exam did not find any cog-wheeling, rigidity, shuffling gait, or gait instability. Mental status exam revealed appearing younger than her stated age, anxious mood and flat affect, and thought content revealing the belief that "there are migrant French-Canadian workers at home threatening to hurt my family members." She had seen them at least twice or thrice.

Her Montreal Cognitive Assessment (MOCA) score was 20/30 (score breakdown: $3 / 5$ on visuospatial/executive: missed trails and did not get hands of clock correct; attention: 5/6: missed serial 7 subtraction; language: $2 / 3$ : did not repeat sentences perfectly with regard to pronouns, singular versus plural; delayed recall: $0 / 5$, but recalled all five with category cuing; orientation: 5/6. Her PHQ-9 [4] and GAD-7 [5] scores were 7 and 9 , resp.).

It was thought that it would be exceedingly rare, although not impossible, for the patient to have a new-onset psychosis at this age. However, the patient's description of her symptoms was not classically Charles Bonnet syndrome, as she experienced "real people talking and interacting" in addition to just seeing them. We decided to re-do MRI and obtain dementia workup, including B12/folate, and other basic labs, which were all unremarkable.

At our second appointment several months later, we again noticed the constellation of visual hallucinations, cognitive impairment, and history of multiple falls despite not having any clear Parkinsonian features on physical exam. At this appointment, both the patient and her family were more significantly distressed by especially the auditory hallucinations component of her experiences, described as "little people threatening to do things to me or my family." Her PHQ-9 and GAD-7 scores had worsened to 10 and 17, respectively. MRI 
was unchanged compared to a year ago. It was thought at this time that the patient's distressing symptoms as well as psychotic symptoms could benefit from trial of quetiapine, to which the patient was naïve. Concurrently, we aimed to decrease clonazepam dosage from $3 \mathrm{mg}$ to $2 \mathrm{mg}$ per day over several weeks.

At our 3rd appointment, a month later, the patient reported that "everybody is noticing the difference with the Seroquel (quetiapine)." In fact, she described no longer having any AVH. Her quetiapine dosage had been titrated to effect to $200 \mathrm{mg}$ PO QHS. Her PHQ-9 and GAD-7 scores were both zero. Of particular significance was also that the patient had tapered off clonazepam completely. As a result, both her headaches and her memory complaints had also decreased. The patient stated that since she was doing so well, she declined formal MOCA retesting.

However, at our 4th appointment, two months since the previous one, the patient described having horrible headaches and having hallucinations again, described as "I see people in the bathroom dressed in regular clothes, and they just stand there without talking to me." The patient and her family did not seem to think that her headaches and hallucinations were connected. She also stated that sleep was awful despite taking hydroxyzine, melatonin, and quetiapine concurrently. We thus decided to transition her sertraline to venlafaxine using cross-titration.

At our 5th appointment a month and a half later, the patient had in the interim requested increasing quetiapine from 200 to $300 \mathrm{mg}$ PO at night, and she was noticeably improved with both increase of quetiapine and transition from sertraline to venlafaxine. In fact, her headaches were "now gone completely" and her mood symptoms were also improved: "The new medication is a happy pill!" The patient described having minimal auditory and visual hallucinations which were "no longer bothering me."

Approximately 8 months following her initial presentation, the patient described "things as going very well": she was no longer waking up in the morning with any headaches and felt comfortable with seeing "little happy faces at night" when either falling asleep or waking up. At this point, the patient's venlafaxine dosage was $225 \mathrm{mg}$ PO QDay and quetiapine dosage was between 300 and $400 \mathrm{mg}$ PO at night. Given the patient's historical lack of depth perception and known history of floaters, it was thought that Charles Bonnet syndrome could be considered due to response of patient's AH to quetiapine but not her visual hallucinations. The patient was educated about the possibility that she had Charles Bonnet syndrome in addition to meeting criteria for major depressive disorder with Psychotic Features and she was reassured.

Ten to twelve months following her initial presentation, the patient described having a significant improvement in mood symptoms, and clinical exam and family collateral information indicated her Major Depression was in remission. The patient's medication dosages of venlafaxine $225 \mathrm{mg}$ daily and $400 \mathrm{mg}$ quetiapine at night were stable and did not cause any noticeable adverse effects, and discussion about eventually trying to find the minimally effective doseespecially in the case of the latter medication, due to concerns for potential metabolic and extrapyramidal adverse effects-ensued, although the patient and her family wanted to continue the current dosage given lack of any psychiatric symptoms.

\section{Discussion}

Our differential diagnosis was quite broad and included epilepsy, nonepileptic seizures (NES), depression, anxiety, pseudodementia, mild cognitive impairment/Alzheimer's dementia, Lewy-Body dementia (LBD), Parkinson's dementia, formal thought disorder/schizophrenia, headache, and Charles Bonnet syndrome.

The patient's history of documented NES most likely ruled out the development of new-onset epilepsy at the age of 70 , although partial complex seizures could possibly account for her history of falls. However, the patient and her family's description of her NES made partial complex seizures less likely.

In line with the conceptualization of NES, there is a possibility that the patient's episodes of confusion and wandering alongside the feeling of unfamiliarity of her house might represent dissociative phenomena, specifically depersonalization, which may be associated with psychosocial stressors, although the patient was not able to clearly identify any known stressors.

When the patient had MOCA scores completed, pseudodementia was initially considered given severity of documented depression and anxiety. However, the patient's ability to perform the majority of her ADLs and IADLs as well as having no clinically apparent anomia, aphasia, or apraxia made mild cognitive impairment or Alzheimer's less likely.

Once the patient was found to have memory and cognitive impairment alongside fall frequency, the diagnosis of Lewy-Body Dementia was then considered. However, of the clinical symptomatic triad of fluctuating cognitive impairment, extrapyramidal features, and visual hallucinations, the patient primarily had only the latter once her clonazepam was tapered and discontinued, making LBD less likely. Furthermore, the patient did not demonstrate any extrapyramidal symptoms or clinical sensitivity to quetiapine, an atypical antipsychotic, which definitively goes against the diagnosis of LBD.

The patient's history of frequent falls and residual daytime grogginess was most likely attributable to her usage of nighttime clonazepam. Her history of myriad falls was initially thought to be due to a combination of either NES or syncope, both previously diagnosed, and thus their attribution to Parkinsonism was thought less likely. Much less likely was that the patient presented with 1st onset formal thought disorder at the age of 70 .

The patient's once or twice monthly to near daily chronic headaches or transformed migraines were associated with "flashing lights," and so the notion that her vision was compromised at baseline ("problems with depth perception") was not on the forefront of our conceptualization towards the possibility of Charles Bonnet Syndrome until much later in her clinical course. 


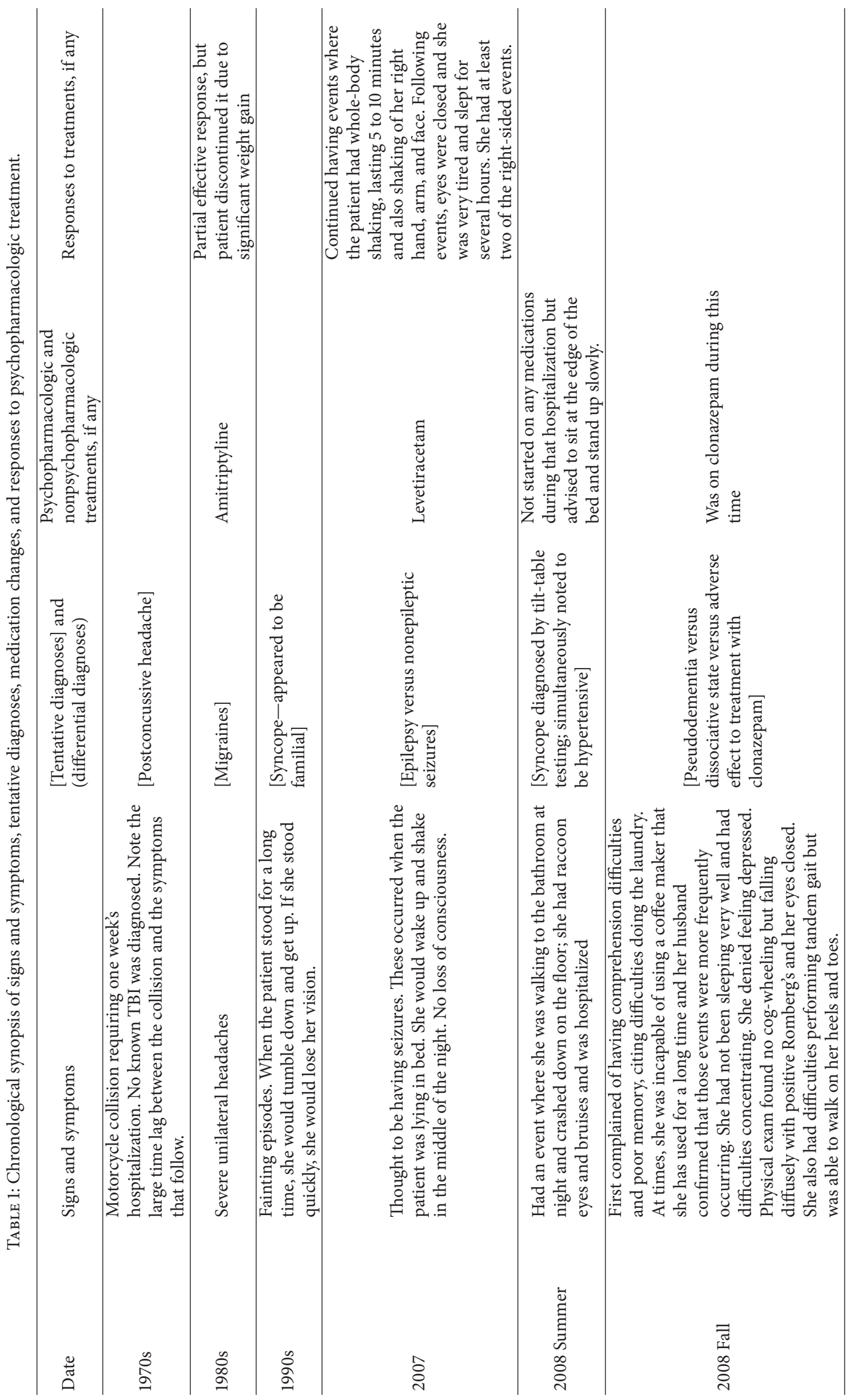




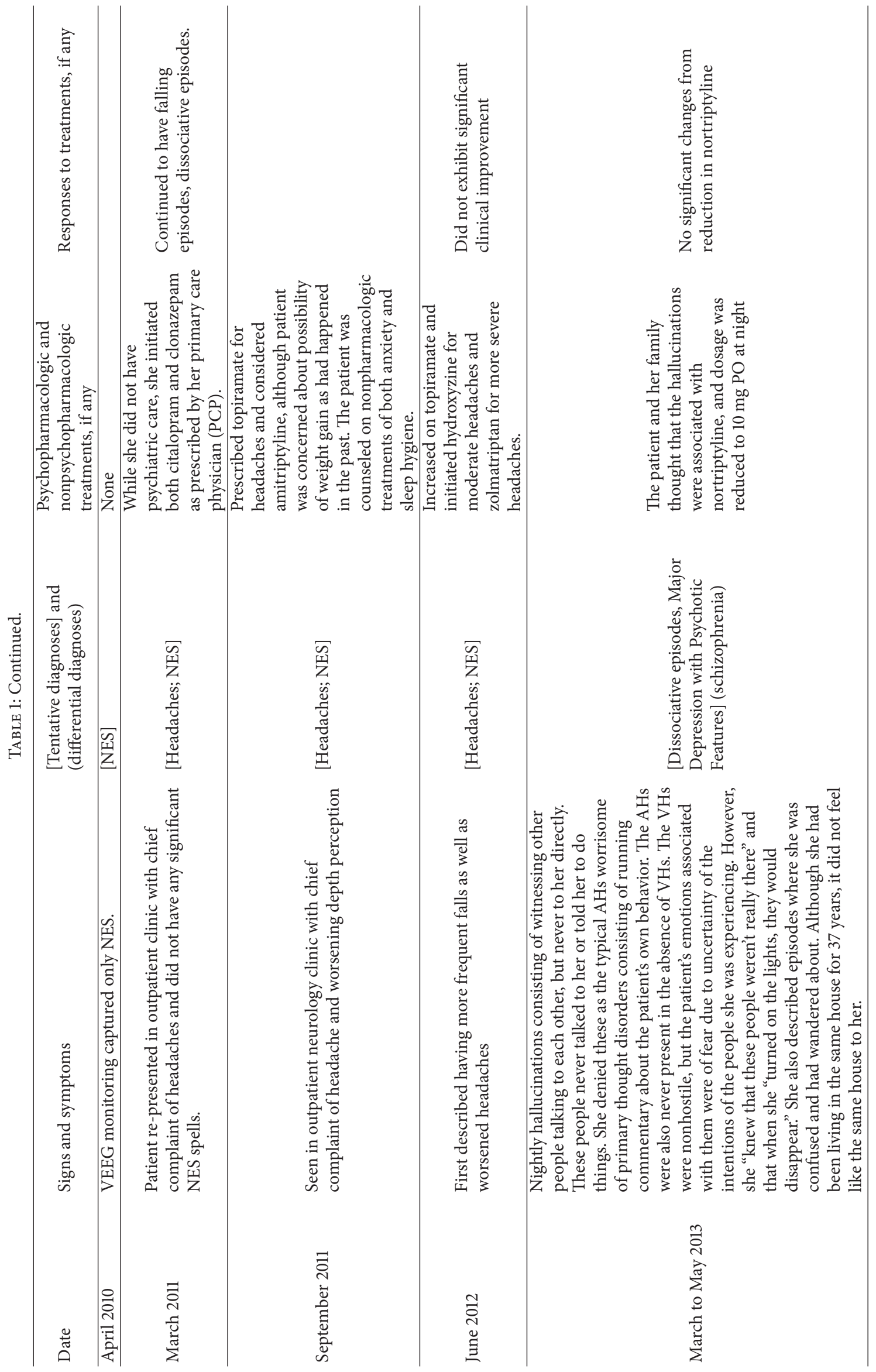




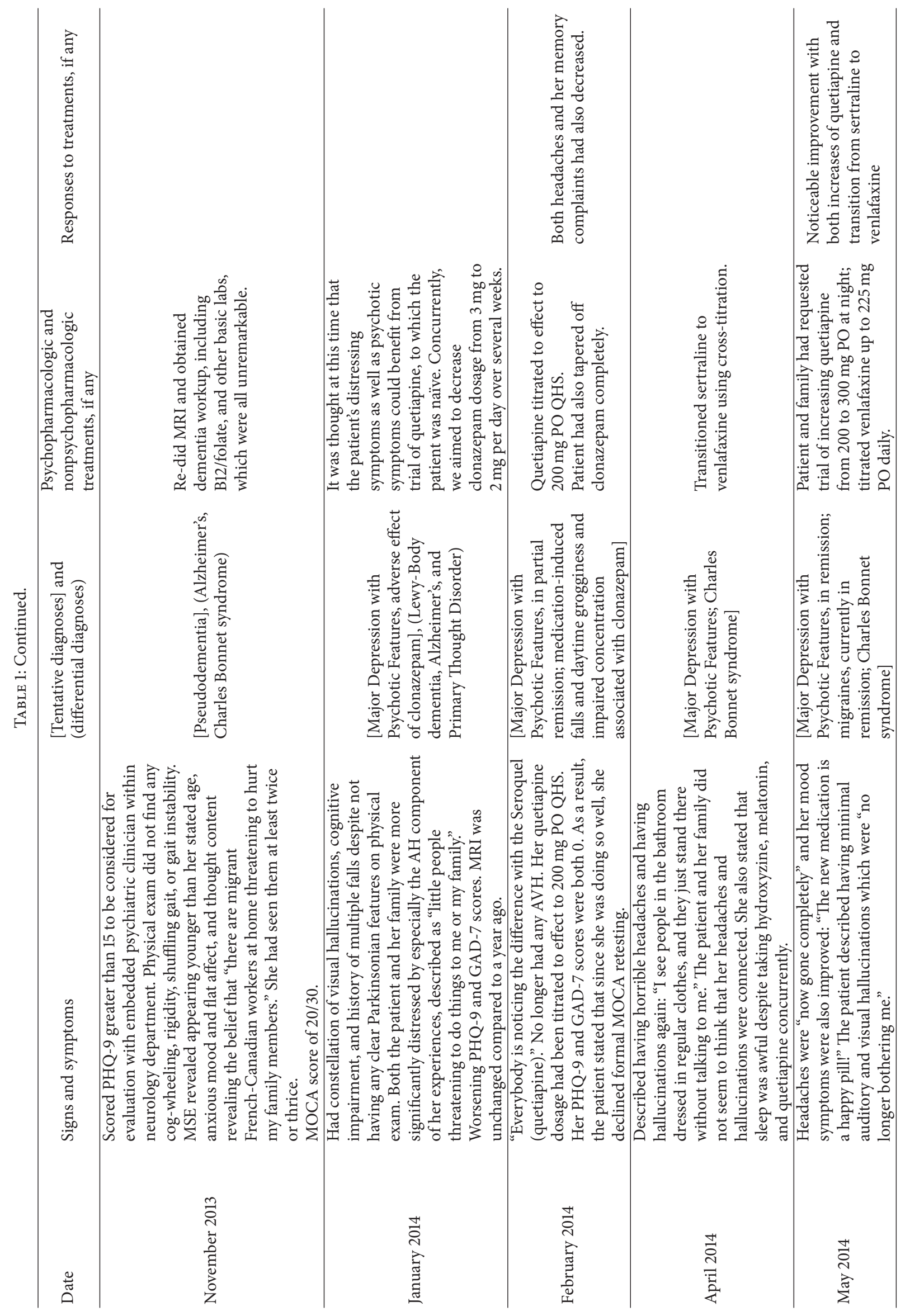




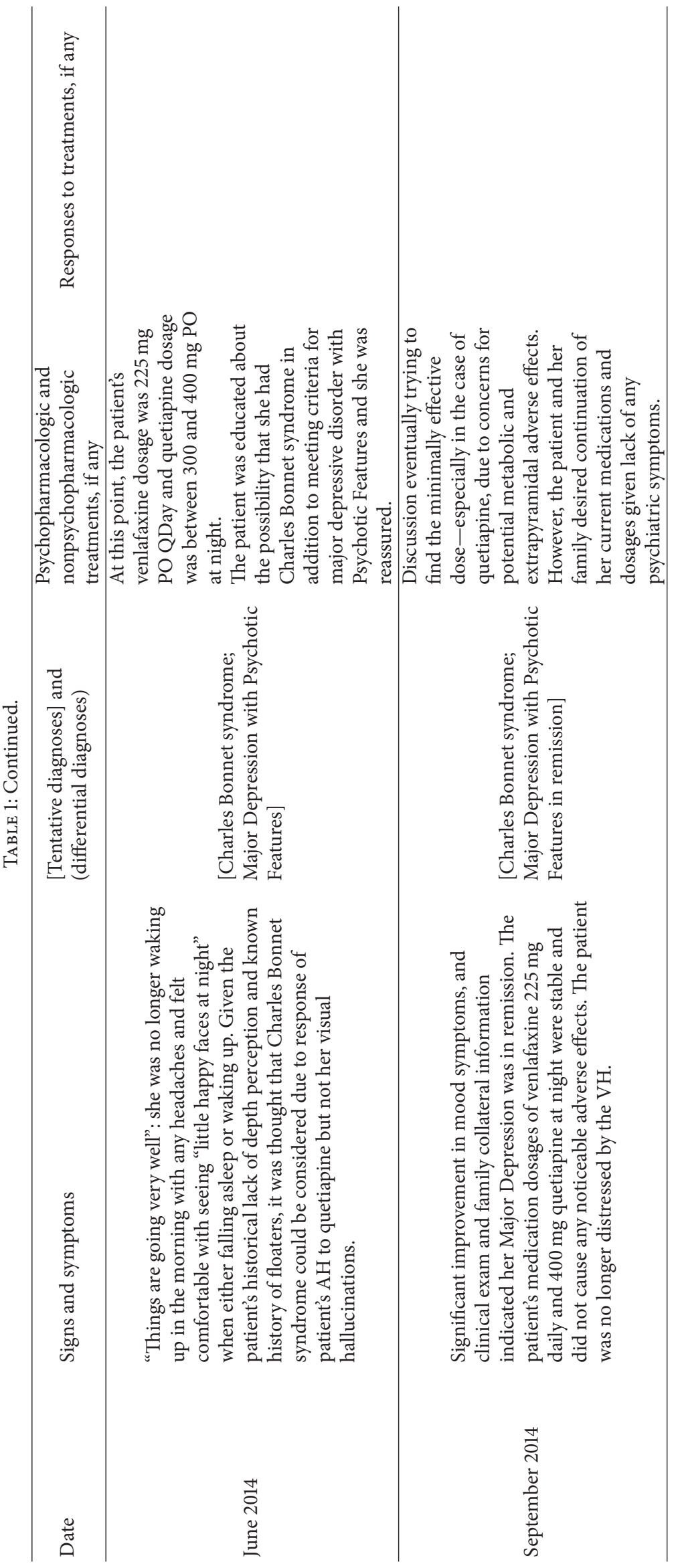


The patient's comorbid mood and anxiety symptoms as well as her preexisting diagnosis of NES made her psychiatric diagnostic formulation particularly challenging. Furthermore, while paranoid delusions are very rarely encountered in patients with CBS, they have been known to be associated with the visual hallucinations experienced [6]. In our patient who was eventually tentatively diagnosed with several comorbid neurological diagnoses-including headache, nonepileptic seizures, and Charles Bonnet syndrome-attributing her psychiatric symptomology parsimoniously to her known neurological diagnoses provided context for psychopharmacologic treatment.

It was thought that the patient's cognitive status, auditory hallucinations, and paranoid ideation were unlikely attributable to simply a diagnosis of Charles Bonnet syndrome. Rather, these clinical features indicated the severity of her psychiatric symptoms such that the patient met diagnostic criteria for major depressive disorder with Psychotic Features.

Our case therefore illustrates the evolution of a patient's symptomology and ultimate benefit of formal psychiatric management. Specifically, venlafaxine ameliorated our patient's anxiety, depression, and headache-whether FDAapproved (anxiety and depression) or off-label (in the case of headache). Quetiapine targeted the amelioration of her anxiety and AVH. Equally noteworthy was that the patient exhibited dramatic improvement following taper and eventual discontinuation of clonazepam.

Given that the medication received by the patient was complex and evolved, please refer to Table $1-\mathrm{a}$ chronological synopsis of signs and symptoms, tentative diagnoses, medication changes, and responses to psychopharmacologic treatment-in order to clarify any drug-related side-effects.

\section{Conclusion}

Referral of this patient with complex neurological history to a co-located psychiatrist within the neurology department guided the diagnostic formulation and eventual diagnoses of the patient's underlying medical and psychiatric comorbidities. Therefore, optimal evaluation and management of mental illness in patients with complex neurological symptoms may require independent treatment by psychiatrists when clinically appropriate. Furthermore, psychiatric comorbidities of patients with neurological disorders may be more optimally addressed by dedicated psychiatrists colocated within neurology clinics in order to reduce utilization and prevent avoidable reencounters $[7,8]$. We advocate for more standardized referral procedures including baseline and longitudinal screening of psychiatric comorbidity using validated instruments for patients seen in neurology clinics.

\section{Authors' Contribution}

Jasper J. Chen oversaw the case report in its entirety and is fully responsible for content.

\section{References}

[1] R. J. Teunisse, J. R. Cruysberg, W. H. Hoefnagels, A. L. Verbeek, and F. G. Zitman, "Visual hallucinations in psychologically normal people: charles Bonnet's syndrome," The Lancet, vol. 347, no. 9004, pp. 794-797, 1996.

[2] A. P. Schadlu, R. Schadlu, and J. B. Shepherd III, "Charles Bonnet syndrome: a review," Current Opinion in Ophthalmology, vol. 20, no. 3, pp. 219-222, 2009.

[3] G. J. Menon, I. Rahman, S. J. Menon, and G. N. Dutton, "Complex visual hallucinations in the visually impaired: the Charles Bonnet syndrome," Survey of Ophthalmology, vol. 48, no. 1, pp. 58-72, 2003.

[4] K. Kroenke, R. Spitzer, and J. Williams, "The PHQ-9: validity of a brief depression severity measure," Journal of General Internal Medicine, vol. 16, no. 9, pp. 606-613, 2001.

[5] R. L. Spitzer, K. Kroenke, J. B. W. Williams, and B. Löwe, "A brief measure for assessing generalized anxiety disorder: the GAD7," Archives of Internal Medicine, vol. 166, no. 10, pp. 1092-1097, 2006.

[6] C. Makarewich and D. A. West, "Charles bonnet syndromeinduced psychosis? visual hallucinations with paranoid delusions in a visually-impaired man," Journal of Neuropsychiatry and Clinical Neurosciences, vol. 23, no. 4, pp. 6-15, 2011.

[7] T. A. Caller, J. J. Chen, J. J. Harrington, K. A. Bujarski, and B. C. Jobst, "Predictors for readmissions after video-EEG monitoring," Neurology, vol. 83, no. 5, pp. 450-455, 2014.

[8] J. Chen, T. Caller, J. Mecchella et al., "Reducing severity of comorbid psychiatric symptoms in an epilepsy clinic using a colocation model: results of a pilot intervention," Epilepsy Behavior, vol. 39, pp. 92-96, 2014.

\section{Conflict of Interests}

The author declares that there is no relevant financial, ethical, or professional conflict of interests. 


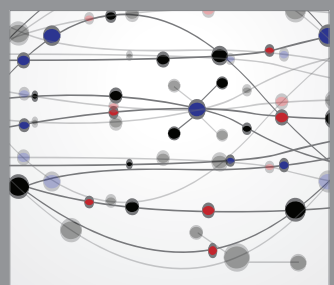

The Scientific World Journal
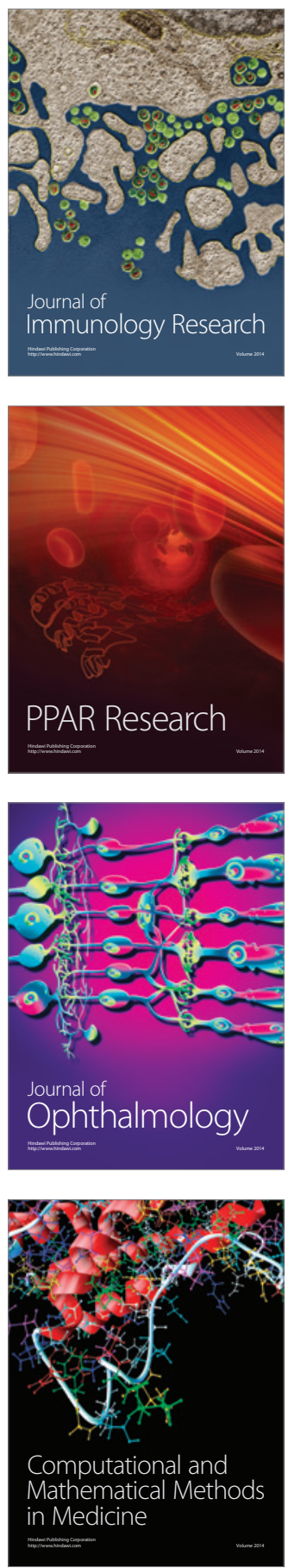

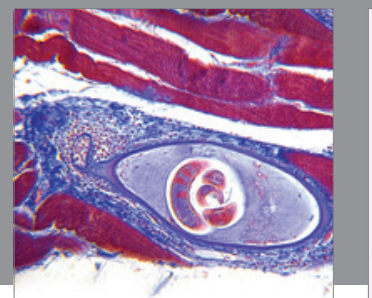

Gastroenterology

Research and Practice
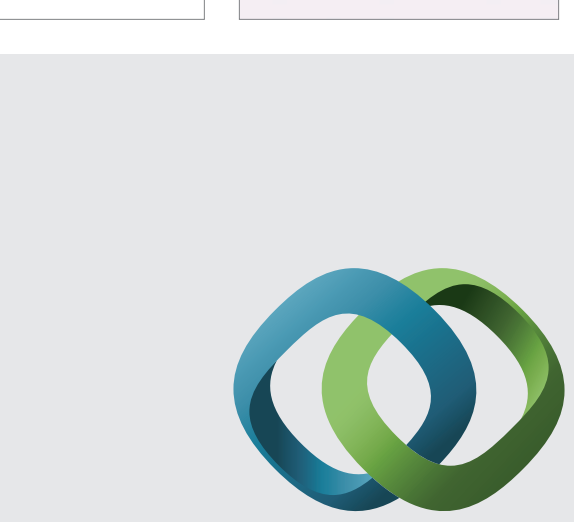

\section{Hindawi}

Submit your manuscripts at

http://www.hindawi.com
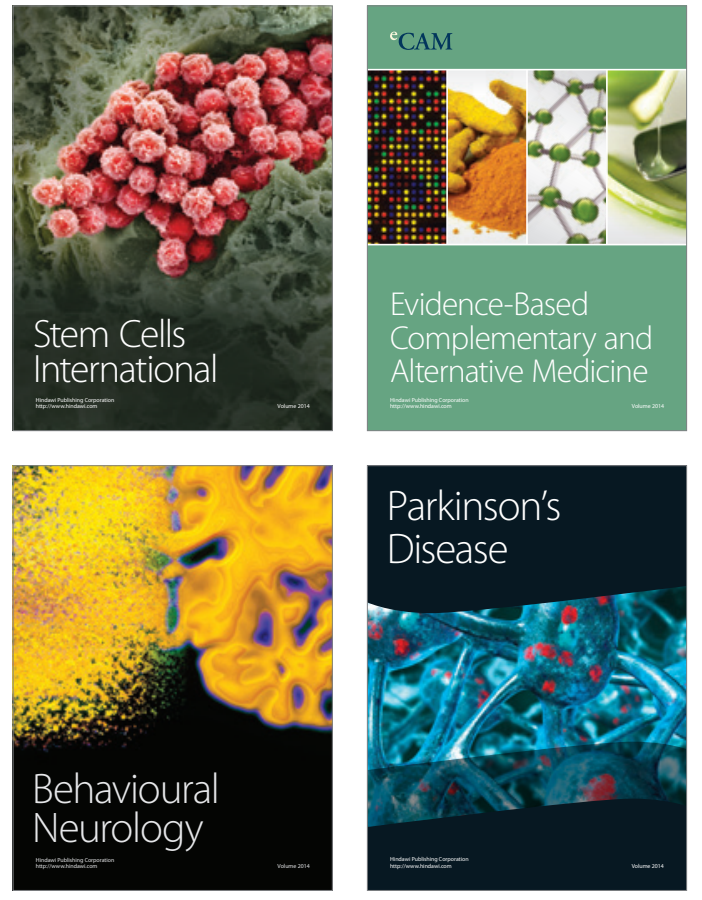
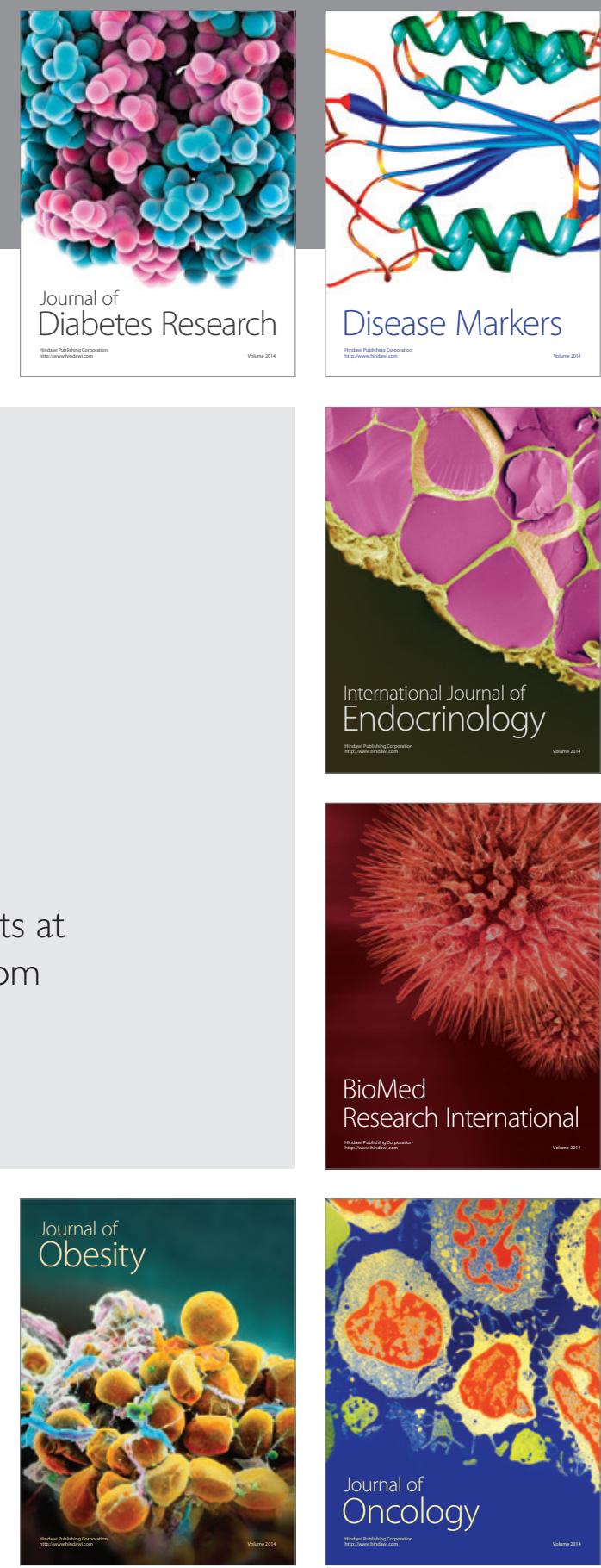

Disease Markers
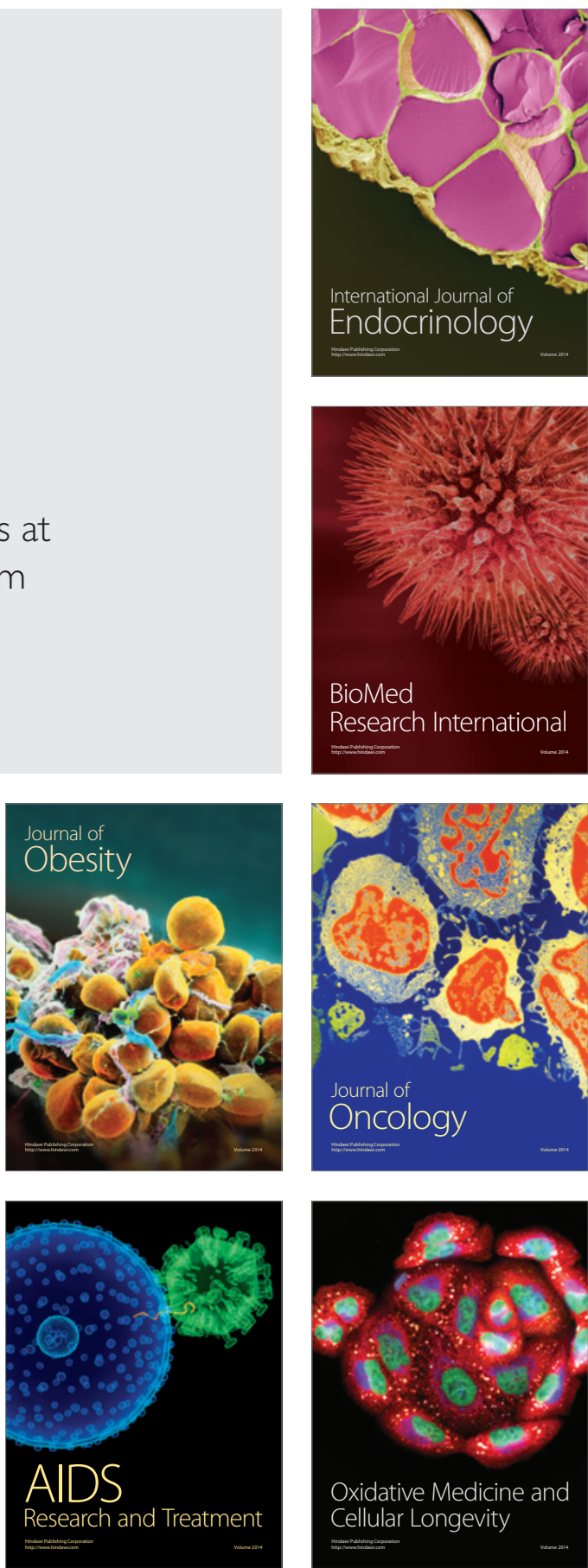